\title{
Projection of Inclusive Search for Gluinos and Squarks at the HL-LHC ATLAS experiment
}

\section{Moe Wakida*}

Nagoya University, Japan

E-mail: wakidadhep1.phys.nagoya-u.ac.jp

\begin{abstract}
Supersymmetry is one of the most promising theories beyond the Standard Model and can solve the Hierarchy problem. For more sensitive searches of such New Physics and more precise measurements of Higgs couplings, the High Luminosity LHC (HL-LHC) experiment [W] is planned to start in 2026, which it is expected to acquire $3000 \mathrm{fb}^{-1}$ of proton-proton collision data at a center-of-mass energy of $14 \mathrm{TeV}$. The ATLAS detector will be upgraded in order to handle higher luminosities. In 2018, groups in the ATLAS and CMS experiments study projections of main physics analysis channels toward the HL-LHC experiment. In particular, we focus on stronglyproduced supersymmetric particles - gluinos and squarks.
\end{abstract}

The 4th KMI International Symposium (KMI2019)

18-20, February 2019

Nagoya, Japan

${ }^{*}$ Speaker. 


\section{Introduction}

Supersymmetry (SUSY) is a generalization of space-time symmetries that predicts new bosonic partners for the fermions and new fermionic partners for the bosons of the Standard Model (SM). If R-parity is conserved, SUSY particles are produced in pairs, and the lightest supersymmetric particle (LSP) is stable and represents a possible dark-matter candidate. The large cross sections predicted for the strong production of supersymmetric particles make the production of gluinos and squarks, which are the fermionic partners of gluons and the scalar partners of first- and secondgeneration quarks, a primary target in searches for SUSY in proton-proton $(p p)$ collisions. Gluinos and squarks can decay through $\tilde{g} \rightarrow q \bar{q} \tilde{\chi}_{1}{ }^{0}$ and $\tilde{q} \rightarrow q \tilde{\chi}_{1}{ }^{0}$ directly to the lightest neutralino, $\tilde{\chi}_{1}{ }^{0}$, assumed to be the LSP (Figure $\mathbb{W}$ ). These final states have no lepton, where a lepton represents an electron or a muon, at least two jets and missing transverse momentum $\left(E_{\mathrm{T}}^{\mathrm{miss}}\right)$. This paper describes the projection of inclusive search for gluinos and squarks at the HL-LHC ATLAS experiment, which is assumed to have an integrated luminosity of $3000 \mathrm{fb}^{-1}$ at a center-of-mass energy, $\sqrt{s}$, of $14 \mathrm{TeV}$ with an average number of interactions per bunch crossing, $\langle\mu\rangle$, of 200 .

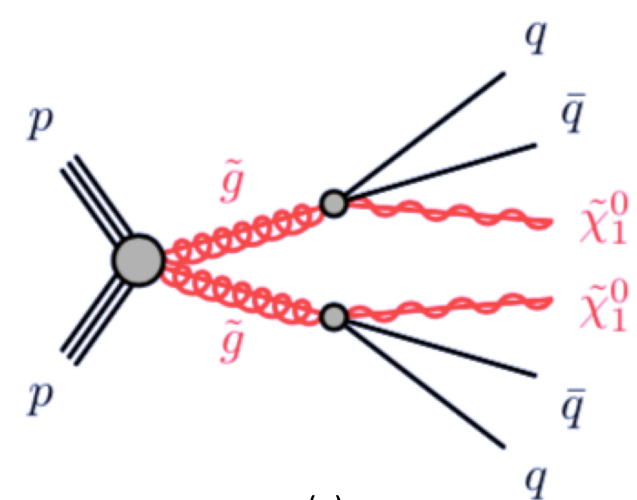

(a)

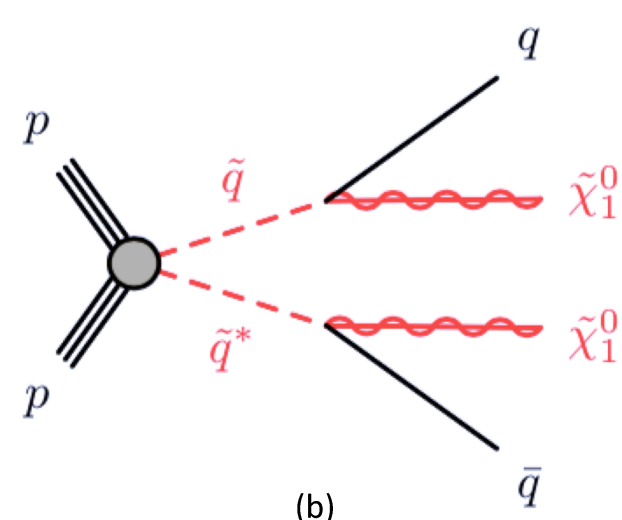

(b)

Figure 1: Decay topology of (a) gluino pair production and (b) squark pair production in the simplified models with direct decays of gluinos and squarks.

\section{Technical setup of the sensitivity projection}

This analysis is based on the method of the previous Run2 analysis with $36 \mathrm{fb}^{-1}$ for the same final states [0]. The effects of an upgraded ATLAS detector are taken into account by applying energy smearing, efficiencies and fake rates to truth-level quantities, following parameterizations based on detector performance studies with the detailed simulations assuming high pile up $\langle\mu\rangle$ $=200$. The number of events at the HL-LHC is estimated from the number of Monte-Carlo (MC) events scaled to $3000 \mathrm{fb}^{-1}$. Almost all the MC samples are generated at $\sqrt{s}=13 \mathrm{TeV}$ and these cross sections are reweighed at $\sqrt{s}=14 \mathrm{TeV}$ considering to parton luminosities. Only the $t \bar{t}$ sample is generated at $\sqrt{s}=14 \mathrm{TeV}$. We follow the recommendations for the treatment of systematic uncertainties expected at the HL-LHC. For example, data-based statistics-driven sources are scaled with 
$\sqrt{L}$, where $L$ is integrated luminosity, theory modeling uncertainties are halved, and uncertainties from the analysis methods are kept the same value as in the previous analysis.

\section{Analysis method}

Main discriminating variables are the number of jets (Njets) and effective mass ( $m_{\mathrm{eff}}(\mathrm{incl}$.)), which is scalar sum of transverse momenta of all jets with $p_{\mathrm{T}}>50 \mathrm{GeV}$ and $E_{\mathrm{T}}^{\text {miss }}$, hence it corresponds to the mass of produced SUSY particles. If produced particles are heavier, $m_{\mathrm{eff}}(\mathrm{incl}$.) is larger. Due to the high mass scale expected for the SUSY models considered in this study, $m_{\text {eff }}$ (incl.) cuts should be tighter to improve sensitivity in the high mass regions of gluinos and squarks. We optimize Signal Regions (SRs) used in the previous Run2 analysis by choosing $m_{\text {eff }}$ (incl.) cuts for the high mass regions of gluinos and squarks. For SR optimization, we use three benchmark signal points for gluinos and squarks respectively; $m\left(\tilde{g}, \tilde{\chi}_{1}^{0}\right)=(1400,1000),(2600$, 1000), (2600, 0) GeV and $m\left(\tilde{q}, \tilde{\chi}_{0}^{1}\right)=(900,700),(1500,700),(2000,0) \mathrm{GeV}$. These points are outside of the expected exclusion region when Run2 SRs are used. We scan $m_{\text {eff }}$ (incl.) cuts by $200 \mathrm{GeV}$ step. We choose the $m_{\text {eff }}$ (incl.) cuts with the highest sensitivity in the cut scans. The $m_{\text {eff }}$ (incl.) cuts where the number of signal MC events is less than 10 are ignored in order to avoid possible fluctuation of statistics. Expected $m_{\text {eff }}$ (incl.) distributions employed in particular for the high mass region of gluinos and squarks are shown in Figure $\square$. We add four new SRs as shown in Table I. The other variable cuts except for $m_{\mathrm{eff}}$ (incl.) are kept to be the same values as those in the previous Run2 analysis. $Z+$ jets, $W+$ jets, top quark pairs, and di/tri bosons are considered as main backgrounds. Table $\square$ shows the expected number of events in the new SRs. Following the recommendation for predicting systematic uncertainties at the HL-LHC, we assume 2-14\% systematic uncertainties on the background yields, depending on SRs.

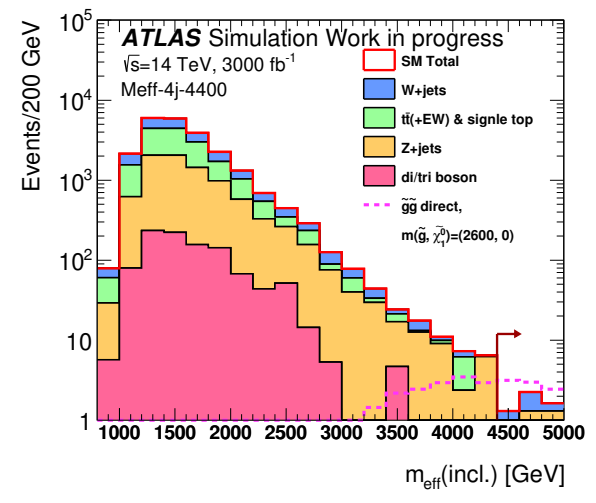

(a)

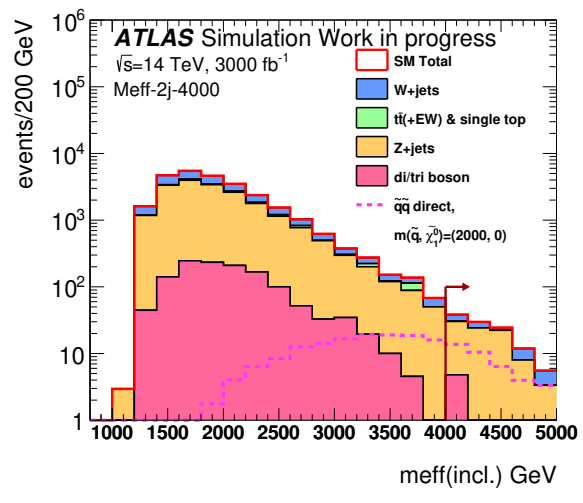

(b)

Figure 2: Expected $m_{\mathrm{eff}}$ (incl.) distributions for the two SRs targeting on the high mass region of (al) gluino and (D) squark with all the selections applied except for the $m_{\text {eff }}$ (incl.) cuts. The red arrows indicate the values at which the requirements on $m_{\mathrm{eff}}$ (incl.) are applied for the new SRs.

\section{Results}

The expected exclusion sensitivity is evaluated by performing a profile-likelihood fit [ [ $]$ ]. For 
Table 1: New selection criteria and targeted signal models added by SR optimization.

\begin{tabular}{|c|c|c|c|c|}
\hline Targeted signal & \multicolumn{3}{|c|}{$\tilde{g} \tilde{g}, \tilde{g} \rightarrow q \bar{q} \tilde{\chi}_{1}^{0}$} & $\tilde{q} \tilde{q}, \tilde{q} \rightarrow q \tilde{\chi}_{1}^{0}$ \\
\hline \multirow{2}{*}{ Requirement } & \multicolumn{4}{|c|}{ Signal Region [Meff-] } \\
\hline & $4 \mathrm{j}-2800$ & $4 j-3400$ & $4 \mathrm{j}-4400$ & $2 j-4000$ \\
\hline$E_{\mathrm{T}}^{\operatorname{miss}}[\mathrm{GeV}]>$ & \multicolumn{4}{|c|}{250} \\
\hline$p_{\mathrm{T}}\left(j_{1}\right)[\mathrm{GeV}]>$ & \multicolumn{3}{|c|}{200} & 350 \\
\hline$p_{\mathrm{T}}\left(j_{2}\right)[\mathrm{GeV}]>$ & \multicolumn{3}{|c|}{150} & 350 \\
\hline$p_{\mathrm{T}}\left(j_{4}\right)[\mathrm{GeV}]>$ & \multicolumn{3}{|c|}{150} & - \\
\hline$\left|\eta\left(j_{1,2,3,4}\right)\right|<$ & \multicolumn{3}{|c|}{2.0} & - \\
\hline$\Delta \phi\left(\text { jet }_{1,2,(3)}, \vec{E}_{\mathrm{T}}^{\text {miss }}\right)_{\min }>$ & \multicolumn{3}{|c|}{0.4} & 0.8 \\
\hline$\Delta \phi\left(\text { jet }_{i>3}, \vec{E}_{\mathrm{T}}^{\text {miss }}\right)_{\min }>$ & \multicolumn{4}{|c|}{0.4} \\
\hline$E_{\mathrm{T}}^{\text {miss }} / m_{\text {eff }}\left(N_{\mathrm{j}}\right)>$ & \multicolumn{3}{|c|}{0.2} & - \\
\hline$E_{\mathrm{T}}^{\text {miss }} / \sqrt{H_{\mathrm{T}}}\left[\mathrm{GeV}^{1 / 2}\right]>$ & \multicolumn{3}{|c|}{-} & 18 \\
\hline Aplanarity > & \multicolumn{3}{|c|}{0.04} & - \\
\hline$m_{\mathrm{eff}}($ incl. $)[\mathrm{GeV}]>$ & 2800 & 3400 & 4400 & 4000 \\
\hline
\end{tabular}

Table 2: Expected yields for the considered backgrounds and selected signal points normalized to $\int L=3000 \mathrm{fb}^{-1}$ in the newly added SRs.

\begin{tabular}{|l|cccc|}
\hline Signal Region $[$ Meff-] & $\mathbf{4 j - 2 8 0 0}$ & $\mathbf{4 j - 3 4 0 0}$ & $\mathbf{4 j - 4 4 0 0}$ & $\mathbf{2 j - 4 0 0 0}$ \\
\hline Di/tri boson & $10.0 \pm 5.2$ & $4.7 \pm 3.7$ & $0 \pm 0$ & $5.1 \pm 3.7$ \\
$Z / \gamma^{*}+$ jets & $186.6 \pm 9.2$ & $47.1 \pm 4.3$ & $4.5 \pm 1.2$ & $95.2 \pm 5.7$ \\
$W+$ jets & $76.8 \pm 5.7$ & $11.6 \pm 1.5$ & $2.0 \pm 0.6$ & $25.1 \pm 2.2$ \\
$t \bar{t}(+\mathrm{EW})+$ single top & $47.1 \pm 10.9$ & $9.5 \pm 4.9$ & $0 \pm 0$ & $0 \pm 0$ \\
\hline Total background & $320.5 \pm 16.3$ & $72.9 \pm 7.6$ & $6.4 \pm 1.3$ & $125.5 \pm 7.1$ \\
\hline \multirow{2}{*}{ Signal } & $m\left(\tilde{g}, \tilde{\chi}_{1}^{0}\right)=(1400,1000) \mathrm{GeV}$ & $m\left(\tilde{g}, \tilde{\chi}_{1}^{0}\right)=(2600,1000) \mathrm{GeV}$ & $m\left(\tilde{g}, \tilde{\chi}_{1}^{0}\right)=(2600,0) \mathrm{GeV}$ & $m\left(\tilde{q}, \tilde{\chi}_{1}^{0}\right)=(2000,0) \mathrm{GeV}$ \\
\cline { 2 - 5 } & $60.3 \pm 1.4$ & $18.9 \pm 0.1$ & $13.8 \pm 0.1$ & $43.3 \pm 1.3$ \\
\hline
\end{tabular}

each signal point, the $p$-values for the background-only hypothesis $\left(p_{\mathrm{b}}\right)$ and for the signal and background hypothesis $\left(p_{\mathrm{s}+\mathrm{b}}\right)$ are calculated respectively, from the expected numbers of background and signal events. When the ratio of $p_{\mathrm{b}}$ and $p_{\mathrm{s}+\mathrm{b}}$ is less than 0.05 , the point is excluded at $95 \%$ confidence level. Limits are obtained by using the SRs with the best expected sensitivity at each mass point. Figure 3$]$ shows expected exclusion limits and $5 \sigma$ discovery limits for direct production of gluino or first- and second-generation squark pairs. For direct production of gluino pairs and squark pairs, the $95 \%$ confidence level expected limits are set up to $2800 \mathrm{GeV}$ and $2150 \mathrm{GeV}$, and $5 \sigma$ discovery limits are set up to $2400 \mathrm{GeV}$ and $1800 \mathrm{GeV}$ at the HL-LHC ATLAS experiment with $3000 \mathrm{fb}^{-1}$.

\section{Conclusion}

We study projection of inclusive search for gluinos and squarks in final states with jets and missing transverse momentum at the HL-LHC with $3000 \mathrm{fb}^{-1}$. We have optimized SRs incorporating the increased statistics. Expected exclusion limits for direct production of gluino pairs and squark pairs are extended up to $2800 \mathrm{GeV}$ and $2150 \mathrm{GeV}$. 


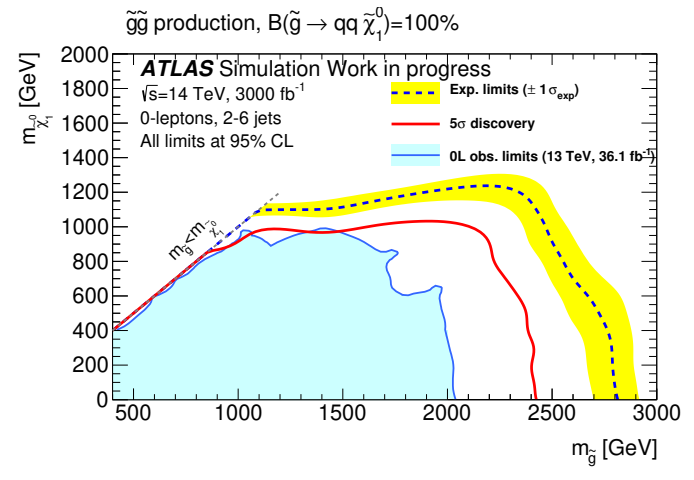

(a)

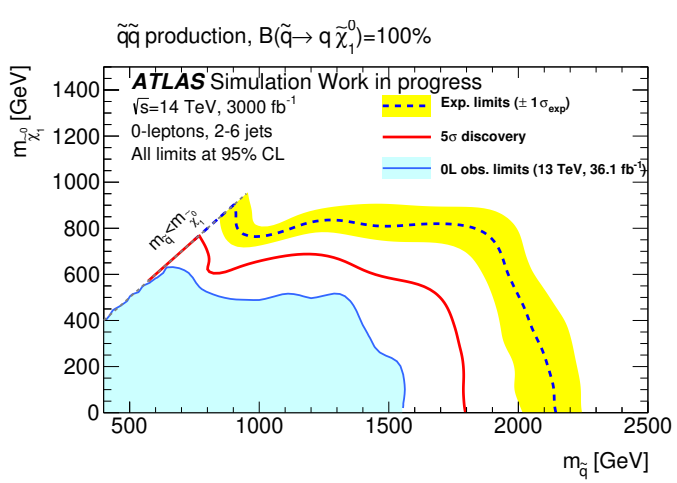

(b)

Figure 3: Expected exclusion and $5 \sigma$ discovery limits for direct production of (⿴囗十) gluino pairs and (B) squark pairs. The blue dash lines show the expected exclusion limits at 95\% CL, with the yellow bands indicating the $\pm 1 \sigma$ systematics uncertainties. The red solid lines show the expected $5 \sigma$ discovery limits. The light blue area shows excluded region by the previous ATLAS Run2 analysis with $36 \mathrm{fb}^{-1}[$ [2].

\section{References}

[1] ATLAS collaboration, Expected performance of the ATLAS detector at the High-Luminosity, ATL-PHYS-PUB-2019-005.

[2] ATLAS collaboration, Search for squarks and gluinos in final states with jets and missing transverse momentum using $36 \mathrm{fb}^{-1}$ of $\sqrt{s}=13 \mathrm{TeV}$ pp collision data with the ATLAS detector, Phys. Rev. D 97,112001 [hep-ex/1712.02332].

[3] Kyle S. Cranmer, Practical Statistics for the LHC, CERN-2014-003, pp. 267 - 308 [physics.data-an/1503.07622]. 